\title{
A simple novel technique [PUIT] for closure of urethrocutaneous fistula after hypospadias repair: Preliminary results
}

\author{
M. S. Awad \\ Department of Plastic Surgery, Zagazig University Hospitals, Cairo, Egypt
}

Address for correspondence: MS Awad, Department of Plastic Surgery, Zagazig University Hospitals,Cairo, Egypt.

E-mail: dadlahm@yahoo.com

\begin{abstract}
Urethrocutaneous fistula is a common complication of urethroplasty for severe hypospadias, even when a microsurgical technique is applied, the closure of the fistula is a challenging problem. We present a simple surgical procedure, posterior urethral incision technique [PUIT] to close the fistula in our department.

Between February 2001 and December 2004 we prospectively evaluated 32 patients, 26 patients with initial hypospadias fistulas and 6 with recurrent fistulas who underwent closure of urethrocutaneous fistula after hypospadias repair. The mean age of patients was 5 years, the operation consisted of trimming the fistula edge after mobilization of the skin all-around then a midline posterior urethral incision was done $2 \mathrm{~mm}$ above and $2 \mathrm{~mm}$ below the fistula opening then re-approximation of the urethral edges using $6 / 0$ vicryl sutures with loop magnification.

The timing of fistula repair was between 6 and 13 months after it was formed, all of these were effectively closed except three cases with stricture and fistula. Of these, two were completely relieved after repeated urethral dilatation, three times a week for 2 weeks. The third failed case will need another sitting after 6 months.

The posterior midline urethral incision gives a good opportunity for repair without tension with a good cosmetic outcome. This may be done under local anesthesia in adults. The procedure is considered simple rapid and easy to be done for variable fistulas types whatever of its site and the age.
\end{abstract}

\section{KEY WORDS}

Hypospadias, Fistula, Posterior urethra incision

\section{INTRODUCTION}

lthough a simple closure of a fistula is easy and not time-consuming it is followed by a significantly higher rate of recurrence than when skin flaps are used. Rotational and advancement flaps are the optimal methods for repairing fistulae after hypospadias, particularly for large and coronal fistulae. However these have a high incidence of recurrence that needs a highly trained surgeon with suprapubic diversion especially in those with large or multiple fistulae. ${ }^{1}$

The effect of suture materials on urethroplasty complications is debatable. Indeed, materials with a delayed 
absorption might either reduce the incidence of fistulas by ensuring a prolonged approximation of neo-urethral edges or increase the risk of urethral strictures due to a prolonged tissue reaction during suture absorption. ${ }^{2}$ The aim of this study is to do a simple easy surgical technique for closure of the fistula whatever its size and site with promising results.

\section{PATIENTS AND METHODS}

In the period between 2001 and 2004, 32 patients with urethrocutaneous fistulas were admitted at our surgical department and repaired under local anesthesia for adult patients and general anesthesia for children. The patients were aged from 2 to 18 years, with a mean age of 5 years. The age of the patients, site, size of the fistula and the preoperative clinical details are listed in Tables 1 and 2 .

Technique: We dissect the skin edge around the urethral fistula $5 \mathrm{~mm}$ all-around [Figures 1, 2 and 3] then trimming the fistula edge after putting the catheter in place, a posterior midline incision $2 \mathrm{~mm}$ above and $2 \mathrm{~mm}$ below is made on the urethral wall [Figure 4]. After the incision is completed the urethral catheter is placed in site and the edge closed in two layers with $6 / 0$ vicryl sutures. The first layer is the urethral edge and the second layer is the dartos fascia [Figure 5].

Table 1: Age at operation $(n=32)$

\begin{tabular}{lccc}
\hline Age group & Age & $\boldsymbol{n}$ & Total \\
$<3$ years & 24 months & 1 & \\
& 26 months & 6 & \\
& 28 months & 4 & \\
30 months & 2 & \\
& 36 months & 2 & 15 \\
$3.5-4$ years & & 9 & 9 \\
$>4$ years & $4-18$ years & 6 & \\
& $5-10$ years & 2 & 8 \\
\hline
\end{tabular}

Table 2: Site and size of the fistula

\begin{tabular}{lcc}
\hline $\begin{array}{l}\text { Fistula characters } \\
\text { Site of the fistulas }\end{array}$ & $\boldsymbol{n}$ & $\%$ \\
$\begin{array}{l}\text { Proximal } \\
\text { Distal }\end{array}$ & 9 & 28.2 \\
Coronal & 10 & 31.2 \\
Type of the fistulas & 13 & 40.6 \\
Primary after Mathieu & & \\
TIP operation & 13 & 40.6 \\
Onlay operation & 8 & 25 \\
Recurrent fistulas & 5 & 15.6 \\
$<3 \mathrm{~mm}$ & 6 & 18.6 \\
$>3 \mathrm{~mm}$ & 18 & 56.3 \\
& 14 & 43.7 \\
\hline
\end{tabular}

\section{RESULTS}

All the patients with primary hypospadias $(n=26)$ patients or recurrent fistulas $(n=6)$ were operated with our technique 6-13 months after hypospadias repair, we noticed that many hypospadias types were coronal at junction between urethra and neourethra $(n=13)$, nearly all the fistulas developed 3-5 days after stent removal (1113 days after hypospadias repair, either TIP, onlay graft or Mathieu operations).

The mean age of the patients was 5 years, the fistula size ranged from 2 to $7 \mathrm{~mm}$. The success of repair was $92.3 \%$ in large fistulas and $94.4 \%$ in small fistulas, with no significant difference between the two types and postoperative strictures were noted in three cases. Successful repair was achieved in 29 patients $(90.6 \%)$. The other three patients had infected wounds with recurrent fistulas, two of them at the junction between the urethra and neourethra and the third at a distal site. Postoperative regular dilatation was done twice weekly for 3 weeks to treat the stricture and close the fistula except in one patient who required another session after 6 months [Table 3]. The follow-up period ranged from 4 to 8 months.

\section{DISCUSSION}

Fistula formation is the commonest complication after hypospadias repair. Surgery for hypospadias fistulas has remained a challenge for the treating surgeons and several surgical procedures have been described each claiming good results. With refined surgical techniques, fine suture materials, and special dressings, the results of surgery after hypospadias repair have improved significantly. ${ }^{3}$ Distal narrowing and infection are important factors to determine

Table 3: The success and failure rates according to the age, size and type of the fistulae

\begin{tabular}{lcccc} 
& \multicolumn{2}{c}{ Success } & \multicolumn{2}{c}{ Stricture } \\
Age & $\mathbf{n}$ & $\%$ & $\mathbf{n}$ & $\%$ \\
$<4$ years & 22 & 91.6 & 2 & 6.3 \\
$>4$ years & 7 & 87.5 & 1 & 3.1 \\
Site & & & & \\
Proximal & 9 & & & \\
Distal & 8 & 80 & 2 & 6.3 \\
Coronal & 12 & 92.3 & 1 & 3.1 \\
Size & & & & \\
$<3$ mm & 17 & 94.4 & 1 & 6.3 \\
$>3$ mm & 12 & 92.3 & 2 & 3.1 \\
Total & 29 & 90.6 & 3 & 9.4 \\
\hline
\end{tabular}


the surgical outcome. ${ }^{4}$ To avoid fistulae after hypospadias repair Yutaro et $a l^{5}$ recommend the use of scrotal dartos flap as a well vascularised and long flap to envelop the neourethra after TIP operation for the prevention of the

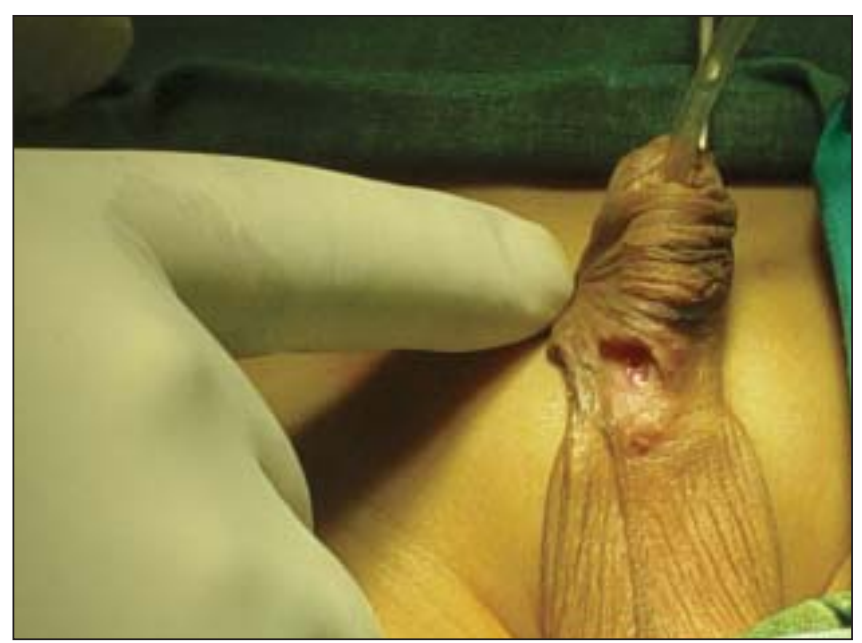

Figure 1: Bilateral fistulas after TIP operation

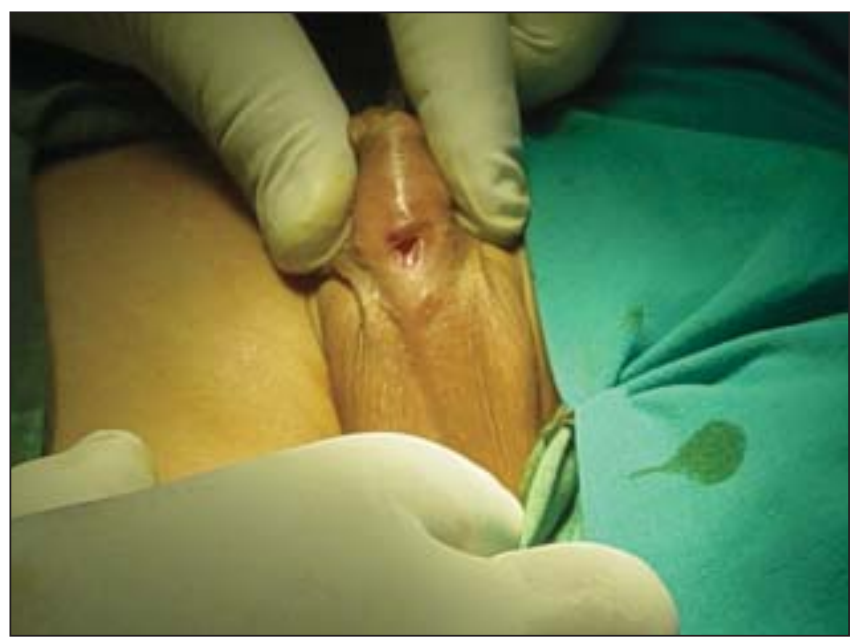

Figure 2: Distal penile fistulas

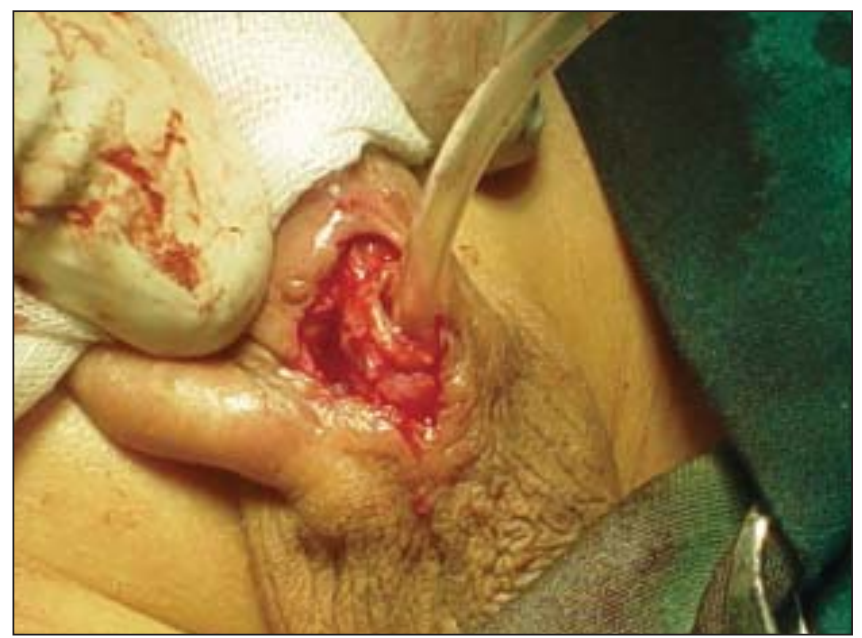

Figure 3: Release of the skin around the fistula urethrocutaneous fistula. Some authors attribute suture materials, appropriate technique, meticulous surgery, and surgeon experience to be more crucial factors, ${ }^{2}$ Cimador $e t$ al suggested that the suture materials do not affect the complication rate in flap urethroplasty procedures. Jan et $a l^{6}$ published that the incidence of fistula formation after hypospadias repair was low if monofilament subcuticular sutures was used.

Several procedures have been used for closure of fistula, including simple closure and complex operations according to the site and size of the fistula. Simple closure is technically easy, but the results are less than promising. ${ }^{7,8}$ In our study, the majority of fistulas occurred at the junction of the neourethra with the urethra, and we close all the fistulas with continuous subcuticular vicryl $6 / 0$ sutures by the same surgeon with good results.

Regarding the time of fistula closure, we prefer to close a

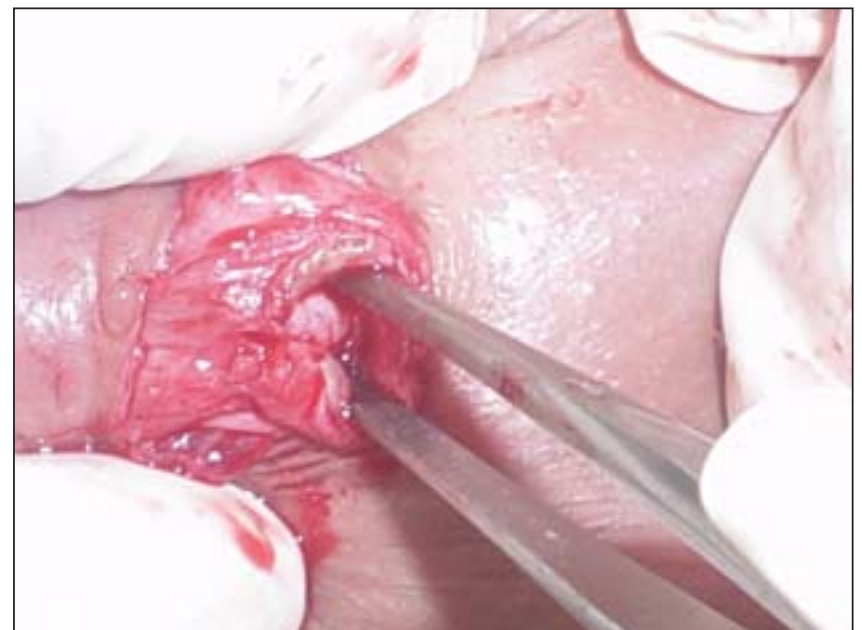

Figure 4: Midline posterior urethral incision

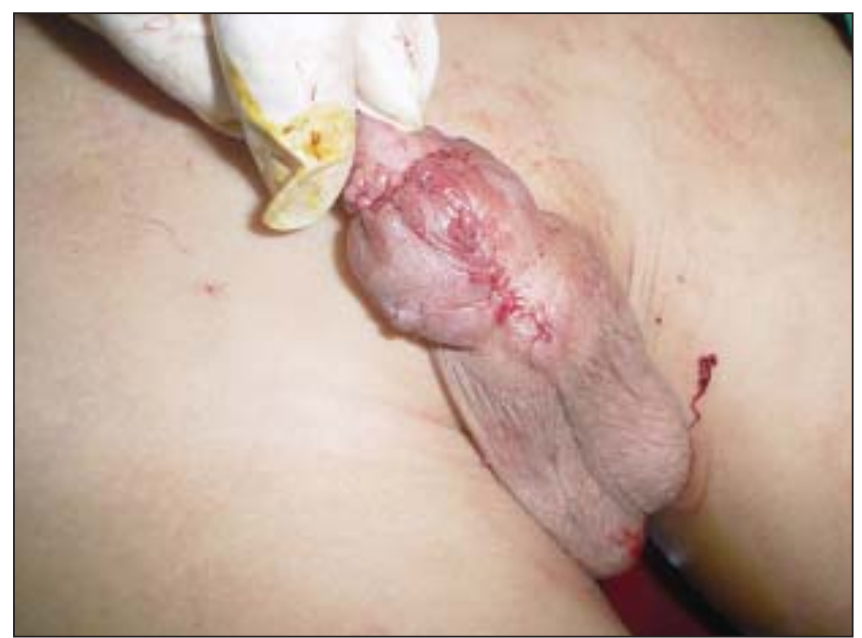

Figure 5: Postoperative picture after double layers closure 
fistula after 6 months to allow induration and scarring to subside. We also wanted to see if some fistulae might close spontaneously in response to regular dilatation. As the distal narrowing is an important factor for fistula formation. ${ }^{4,9}$

Our surgical technique to close the fistula, small or big $(<3$ or $>3 \mathrm{~mm}$ ) is a simple procedure, through posterior midline urethral incision to close the fistula without tension, where tension suture is the cause of failure after any repair, we avoid this tension through the midline posterior incision and adding a vascularized flap over the sutures with the surrounding dartos layer to promote healing and creating a barrier between the skin sutures and the sutures for fistula.The success rate in our study was about (94.4\%) for small fistulae and $85.7 \%$ for large fistulae. Zhao et $a l^{10}$ used double skin flaps or pedicled skin flap from the scrotum for the repair. In the latter cases, transverse $Y$ or $V$ shaped skin flaps were used to close fistulae both small and large with a success rate $87.5 \%$ in small fistulae, and $86.84 \%$ for large fistulae. Other authors ${ }^{11}$ preferred a de-epithelialized or full thickness skin advancement flap for coverage of fistula,the cases closed with a simple technique had a failure rate $4.3 \%$. In 25 patients with failure and who required more complex repairs, 18 underwent a tubularized or onlay urethroplasty incorporating the fistula, which failed in $2(11.1 \%)$.

Essammarzouk $^{12}$ published a technique of fistula advancement for repair of recurrent distal urethrocutaneous following distal penile hypospadias repair with excellent results and he claimed that this operation solve the problem of persistently recurrent hypospadias fistula with a high success rate but it was restricted to the distal penile fistulas. András et $a l^{13}$ stated that in cases with recurrent large fistula after hypospadias reconstruction, the use of buccal mucosa patch graft for closure is a good treatment choice with success of six out of seven cases $(85.7 \%)$. The success rate in our study was (90.6\%).

Regarding the use of stent or microsurgery the data from a study done by Waterman et $a l^{14}$ suggested that there is no clear difference in stent $V s$ no stent and microscope $V s$ loupes, age at fistula closure does not affect success, type of original hypospadias procedure may influence success and success rate is not negatively impacted in recurrent fistula cases. Sahin et $a l^{15}$ reported that the success rate of fistula repair was 93.3 and $94.1 \%$ in the catheterized and uncatheterized groups, respectively. As a result, the authors suggest that catheter less fistula repair with local anesthesia in the adult age group is an effective, safe, and inexpensive procedure.

We catheterized all our patients. We noticed that, the age at fistula closure does not affect success also site of the fistula and type of original hypospadias operation does not influence fistula success. Elbakry, ${ }^{1}$ stated that although simple closure of a fistula is easy and not time-consuming it is followed by a significantly higher rate of recurrence than when skin flaps are used, and he advised the rotational and advancement flaps as the optimal methods for repairing fistulae after hypospadias repair, particularly for large and coronal fistulae. Thus, the appropriate indication for simple closure is small fistulae at the penile shaft. Suprapubic diversion is important in those with large or multiple fistulae.

We have done our technique for both small and large fistulas, primary and recurrent types without suprapubic diversion with the same good results.

\section{CONCLUSIONS}

The posterior urethral incision technique [PUIT] for fistula repair, as it is called by the author, can, solve the problem of hypospadias fistula either primary or recurrent, small or large, distal coronal and proximal, with a high success rate and promising results.

\section{REFERENCES}

1. Elbakry Management of urethrocutaneous fistula after hypospadias repair: 10 years' experience. J Urol 2003;170:328.

2. Cimador M, Castagnetti M, Milazzo M, Sergio M, De Grazia E. Suture materials: do they affect fistula and stricture rates in flap urethroplasties?. J Urol 2002;168:1751-3.

3. Baskin L. Hypospadias. A Critical Analysis Of Cosmetic Outcomes Using Photography. BJU International 2001;87:534-9.

4. Kirkali Z. Tunica Vaginalis: An aid in hypospadias surgery. BJU International 1990;65:530-2.

5. Yutaro H, Yoshiyuki K, Satoshi K, kentaro M, Akihiro N, kenjiro K. Scrotal dartos flap for the prevention of the urethrocutaneous fistula on hypospadias urethroplasty, Int J Urol 2005;12:280.

6. Jan IA, Mirza F, Yaqoot, Ali M, Arian A, Saleem N, Ahmad, Kumar D. Factors influencing the results of Surgery for Hypospadias: Experience at NICH. JPMA 2004;54:577.

7. Shankar KR, Losty PD, Hopper M, Wong L, Rickwood AMK. Outcome of Hypospadias Fistula Repair. BJU International 2002;89:103-5.

8. Demirbilek S, Kanmaz T, Aydin G, Yucesan S. outcome of Onestage Techniques For Proximal Hypospadias Fistula Repair. Urology $2001 ; 58: 267-70$. 
9. Horton CE, Horton CE. Complications Of Hypospadias Surgery. Clin Plast Surg 1988;15:371-9.

10. Zhao GJ, Liu CX, Wu GD, Zhang YG, Ou. Repair of urethral fistula resulting from correction of hypospadias: experience with 56 cases: Di Yi Jun Yi Da Xue Xue Bao. 2004;24:113.

11. Santangelo K, Rushton H, Belman G, Barry A. Outcome analysis of simple and complex urethrocutaneous fistula closure using a de-epithelialized or full thickness skin advancement flap for coverage. J Urol 2003;170:1589-92.

12. Essammarzouk. Fistula advancement operation in the treatment of selected cases of persistently recurrent urethrocutaneous fistula following distal penile hypospadias repair. Int J Urol 1999;6:135.

13. András Kiss, László Pirót, Levente Karsza, Miklós Merksz. Use of Buccal Mucosa Patch Graft for Recurrent Large Urethrocutaneous Fistula after Hypospadias Repair Urologia Internationalis 2004;72:329-31.

14. Waterman BJ, Renschler T, Cartwright PC, Snow BW, DeVries $R$. Variables in successful repair of urethrocutaneous fistula after hypospadias surgery. J Urol 2002;168:726-30.

15. Sahin C, Aksoy Y, Ozbey I, Polat O. Outpatient Urethrocutaneous Fistula Repair With Local Anesthesia in Adult Patients. Ann Plast Surg 2003;50:378-81. 